\title{
RESPONSE OF CERTAIN PHYTOCHEMICAL CONSTITUENTS IN SWEET PEPPER LEAVES TO SOME BIO-STIMULANTS UNDER TWO TYPES OF SALINITY
}

\author{
Arafa,A.A., Khafagy,M.A., Abo-El Kheer,A.M., and M.F. El-Banna \\ Dept. Agric. Bot., Fac. of Agric., Mansoura Univ., El-Mansoura, Egypt
}

E-Mail: dr.arafa50@yahoo.com; khafagy@mans.edu.eg; prof.aboelkheer@gmail.com and El-Banna@mans.edu.eg;

\section{ABSTRACT}

High salinity level of $\mathrm{NaCl}$ and $\mathrm{CaCl}_{2}(4000 \mathrm{mg} / \mathrm{L})$ markedly increased whereas the low levels and its combination decreased electrolyte leakage percentage (E.L. \%) in sweet pepper leaves as compared to the unstressed plants. In addition, application of all selected bio-regulators used at both applied levels, in most cases, alleviated the harmful effect of salinity on E.L. \% especially ascorbic acid at $50 \mathrm{mg} / \mathrm{L}$.

Relative water content (RWC) and photosynthetic pigments were significantly increased thereafter decreased as salinity levels increased. In addition, $\mathrm{CaCl}_{2}$ at low level caused a great increase in $\mathrm{RWC} \%$ followed by $\mathrm{NaCl}+\mathrm{CaCl}_{2}$ and $\mathrm{NaCl}(2000 \mathrm{mg} / \mathrm{L})$. While, pre-soaking seeds in both levels of the applied bio-stimulants caused a significant increase in RWC \% and the photosynthetic pigments concentrations. In addition, AsA at $50 \mathrm{mg} / \mathrm{L}$ or SA at $75 \mathrm{mg} / \mathrm{L}$ was more effective in this respect as compared to the untreated plants.

Ascorbic acid, proline, total phenols as well as total soluble carbohydrates concentrations in sweet pepper shoot were increased with increasing salinity levels from 2000 to $4000 \mathrm{mg} / \mathrm{L}$ of all salinity types. In addition, $\mathrm{NaCl}$ led to a great increase followed by $\mathrm{NaCl}+\mathrm{CaCl}_{2}$ and $\mathrm{CaCl}_{2}$ as compared to the unstressed plants. In addition, pre-soaking seeds in SA, AsA, $\alpha$-tocopherol and yeast extract at both levels increased ascorbic acid, proline, total phenols as well as total soluble carbohydrates concentrations under saline conditions. Moreover, AsA at $50 \mathrm{mg} / \mathrm{L}$ or SA at $75 \mathrm{mg} / \mathrm{L}$ was more effective in this respect as compared with the other treatments.

Keywords: Salinity; Phytochemical constituents; Sweet Pepper

\section{Council for Innovative Research}

Peer Review Research Publishing System

Journal of Advances in Natural Sciences

Vol. 2, No. 2

editorjansonline@gmail.com

www.cirworld.com

83 I $\mathrm{P}$ a g $\mathrm{e}$

October 09,2014 


\section{INTRODUCTION}

Sweet pepper (Capsicum annuum L.) is among the most important crops for the world human nutrition and its fruits have a good nutritional value in respect to antioxidant compounds, such as vitamin $\mathrm{C}$ and carotenoids (Navarro et al., 2006).

It is a moderately-sensitive to salt stress (Lycoskoufis et al., 2005). It cultivated under open field and greenhouses conditions. In Egypt the cultivated area is around 71428.57 Feddan in 2008, yielded 475000 tons (FAO, 2008)* 1 . In addition, productions throughout the world are around over 24 million tons every year (Casado-Vela et al., 2007). Dry land salinity is also an important, and increasing, problem in some areas of the world (Tester and Davenport, 2003). The effect of salinity on plant caused various physiological and biological changes in plants. It damaged photosynthetic components, i.e. lipid peroxidation (Winston, 1990) and injuries to plant metabolism (Meneguzzo and Navarilzzo, 1999) and/or water deficit, ion uptake, salt-specific damages (Cumming and Elliot, 1991) and oxidative stress in plants (Xiong et al., 2002). Salinity also induces water deficit, even in well-watered soils by decreasing the osmotic potential of soil solutes, thus making it difficult for roots to extract water from their surrounding media (Koca et al., 2007; Sankar et al., 2007). Excessive sodium ( $\left.\mathrm{Na}^{+}\right)$inhibits the growth of many salt-sensitive plants and glycophytes, which include most crop plants. High concentrations of salt in soil enhanced generation of reactive oxygen species (ROS) including $-\mathrm{O}^{2}, \mathrm{H}_{2} \mathrm{O}_{2}$, and $-\mathrm{OH}$ (Wang et al., 2008; $\mathrm{Li}, 2009$ ). To prevent damage to cellular components by ROS, plants have developed a complex antioxidant system. Many components of this antioxidant defense system can be found in various sub-cellular compartments (Hernandez et al., 2000).

Nutrient Film Technique (NFT) crop production (commonly known as NFT cropping is air method of growing in which the plants have their roots in a shallow stream of recalculating water in which are dissolved all the elements required. A root mat develops which is partly in the shallow stream of recalculating water and partly above it. Thus the stream is very shallow and the upper surface of the root mat develops above the water, although it is the air. So around the roots which are in the air there is a film nutrient solution, hence its named nutrient film technique (Cooper, 1979).

Therefore, the present investigation was performed to study the effect of different sources of salinity $\left(\mathrm{NaCl}_{1} \mathrm{CaCl}{ }_{2}\right.$ and its combination 1:1) on certain phytochemical constituents of sweet pepper plant. Moreover, it was intended to investigate effects of pre-soaking seeds in some materials such as vitamins (ascorbic acid and a-tocopherol), bio-regulator (salicylic acid) and Yeast extract to alleviate the harmful effects of such salinity types.

\section{MATEREIALS AND METHODS}

An experiment was carried out in the glasshouse of the Agricultural Botany Dept., Fac. of Agriculture, Mansoura Univ. during the growing season of 2008, to study the response certain phytochemical constituents of sweet pepper plant to different sources of salinity i.e. $\mathrm{NaCl}, \mathrm{CaCl}_{2}$ and its combination $(1: 1 \mathrm{w} / \mathrm{w})$; and how to minimize its harmful effects through pre-soaking seeds in vitamins (Ascorbic acid or a-tocopherol) or bio-regulator (Salicylic acid) or Yeast extract.

\section{Plant materials}

The seeds of sweet pepper (Capsicum annuum L. cv. Orlando), a hybrid 'California Wonder' used in this investigation were secured from the Gohara Co. Cairo, Egypt.

\section{Chemicals:-}

1. Vitamins, ascorbic acid Vit. C (AsA) and $\alpha$-tocopherol Vit. E ( $\alpha$-tocopherol) were supplied by Sigma Chemicals Co., USA and used at the concentration of 50 or $100 \mathrm{mg} / \mathrm{L}$ each.

2. Bio-regulator, salicylic acid (SA) (2-hydroxybenzoic acid) was obtained from Sigma Chemicals, Co., USA. and initially dissolved in $100 \mu \mathrm{L}$ dimethyl sulfoxide and used at the concentrations of 75 and $150 \mathrm{mg} / \mathrm{L}$,

3. Yeast extract, active dry yeast (Saccharomyces cervisiae) was applied at the concentration of 1000 or $2000 \mathrm{mg} / \mathrm{L}$.

4. Salts:

4.1. Sodium Chloride $(\mathrm{NaCl})$ from EL-Gomhoria Co., Egypt and was used at the concentrations of 2000 and 4000 $\mathrm{mg} / \mathrm{L}$.

4.2. Calcium Chloride $\left(\mathrm{CaCl}_{2}\right)$ from EL-Gomhoria Co., Egypt and was used at the concentrations of 2000 and $4000 \mathrm{mg} / \mathrm{L}$.

4.3. Their combination, $\mathrm{NaCl}: \mathrm{CaCl}_{2} 1: 1(\mathrm{w} / \mathrm{w})$ was used at the concentrations of 2000 and $4000 \mathrm{mg} / \mathrm{L}$.

\footnotetext{
** FAO: Food and Agriculture Organization of the united nation, Statistical agricultural database sector. www.http:// faostat.fao.org/site/567/
} 
Table (1): The Molarity (Mol), Electrical Conductivity (E.C.) and pH values for different nutrient solutions.

\begin{tabular}{|c|c|c|c|c|c|c|c|c|c|}
\hline \multirow{3}{*}{\begin{tabular}{|l|} 
Nutrient \\
solution \\
(N.S.) mg/L
\end{tabular}} & \multirow[b]{3}{*}{ N.S. } & \multicolumn{2}{|c|}{ N.S.+ $\mathrm{NaCl}$} & \multicolumn{2}{|c|}{ N.S.+ $\mathrm{CaCl}_{2}$} & \multicolumn{4}{|c|}{ N.S. $+\left\{\mathrm{NaCl}+\mathrm{CaCl}_{2}\right\}(1: 1) \mathrm{w} / \mathrm{w}$} \\
\hline & & \multirow{2}{*}{$\begin{array}{l}2000 \\
\mathrm{NaCl}\end{array}$} & \multirow{2}{*}{$\begin{array}{l}4000 \\
\mathrm{NaCl}\end{array}$} & \multirow{2}{*}{$\begin{array}{l}2000 \\
\mathrm{CaCl}_{2}\end{array}$} & \multirow{2}{*}{$\begin{array}{l}4000 \\
\mathrm{CaCl}_{2}\end{array}$} & \multicolumn{2}{|c|}{$2000\left(\mathrm{NaCl}+\mathrm{CaCl}_{2}\right)$} & \multicolumn{2}{|c|}{$4000\left(\mathrm{NaCl}+\mathrm{CaCl}_{2}\right)$} \\
\hline & & & & & & $\begin{array}{l}1000 \\
\mathrm{NaCl}\end{array}$ & $\begin{array}{l}1000 \\
\mathrm{CaCl}_{2}\end{array}$ & $\begin{array}{l}2000 \\
\mathrm{NaCl}\end{array}$ & $2000 \mathrm{CaCl}_{2}$ \\
\hline Mol (M) & $\begin{array}{c}0 \\
\text { (Control) }\end{array}$ & $3.4 \times 10^{-2}$ & $6.9 \times 10^{-2}$ & $2.0 \times 10^{-2}$ & $3.6 \times 10^{-2}$ & $1.7 \times 10^{-2}$ & $0.9 \times 10^{-2}$ & $3.4 \times 10^{-2}$ & $2.0 \times 10^{-2}$ \\
\hline Ec dSm ${ }^{-1}$ & 2.00 & 5.42 & 8.42 & 4.59 & 7.60 & \multicolumn{2}{|c|}{5.08} & \multicolumn{2}{|c|}{8.08} \\
\hline $\mathrm{pH}$ & 5.50 & 5.77 & 5.80 & 5.19 & 5.30 & \multicolumn{2}{|c|}{5.45} & \multicolumn{2}{|c|}{5.34} \\
\hline
\end{tabular}

Table (2): Weights (g) of pure substances to be dissolved in 1000 liters of water to give the theoretically ideal concentrations (Cooper, 1979).

\begin{tabular}{|l|l|l|}
\hline Substance & Formula & Weight \\
\hline Potassium dihydrogen Phosphate & $\mathrm{KH}_{2} \mathrm{PO}_{4}$ & 263 \\
\hline Potassium Nitrate & $\mathrm{KNO}_{3}$ & 583 \\
\hline Calcium Nitrate & $\mathrm{Ca}\left(\mathrm{NO}_{3}\right)_{2} \cdot 4 \mathrm{H} 2 \mathrm{O}$ & 1003 \\
\hline Magnesium Sulphate & $\mathrm{MgSO}_{4} \cdot 7 \mathrm{H}_{2} \mathrm{O}$ & 513 \\
\hline EDTA Iron & $\mathrm{CH}_{2} \cdot \mathrm{N}_{\left.\left(\mathrm{CH}_{2} \cdot \mathrm{COO}\right)_{2}\right]_{2} \mathrm{Fe} \mathrm{Na}}^{79.0}$ \\
\hline Manganous Sulphate & $\mathrm{MnSO}_{4} \cdot \mathrm{H}_{2} \mathrm{O}$ & 6.10 \\
\hline Boric Acid & $\mathrm{H}_{3} \mathrm{BO}_{3}$ & 1.70 \\
\hline Copper Sulphate & $\mathrm{CuSO}_{4} \cdot 5 \mathrm{H}_{2} \mathrm{O}$ & 0.39 \\
\hline Ammonium Molybdate & $\left(\mathrm{NH}_{4}\right)_{6} \mathrm{Mo}_{7} \mathrm{O}_{24} \cdot 4 \mathrm{H}_{2} \mathrm{O}$ & 0.37 \\
\hline Zinc Sulphate & $\mathrm{ZnSO}_{4} \cdot 7 \mathrm{H}_{2} \mathrm{O}$ & 0.44 \\
\hline
\end{tabular}

After soaking, the sterilized seeds $(25$ seeds/dish) were placed in glass Petri dishes $(11 \mathrm{~cm})$ with a double layer of Whatman No. 1 filter paper. The dishes were left in an incubator in the dark for seed germination at $25 \pm 2^{\circ} \mathrm{C}$ and $90 \%$ relative humidity, and then dishes were covered with aluminum foils for darkness. In order to avoid water losses, $5 \mathrm{ml}$ of the nutrient solution were added to Petri dishes, every 5 days. Thiram was added to the solution at a concentration of $2 \%$ $(w / v)$ to control the fungi infection.

The following experiment was carried out in the glasshouse of the Agric. Bot. Dept., Fac. of Agric., Mansoura Univ. during the spring-summer period of 2008 in a glasshouse under conditions of ambient light during winter, spring and early summer, with $10 / 14$ light/dark period at $800-1100 \mu \mathrm{mol} \mathrm{m} \mathrm{m}^{-2 \mathrm{~s}}$ PPFD, a day/night average temperature cycle of $26 / 15^{\circ} \mathrm{C}$ and $65 \pm 5 \%$ relative humidity.

The focus of the current experiment was to provide fundamental biological understanding and knowledge on sweet pepper plants growing in nutrient film technique (NFT), under different sources of salinity $\mathrm{NaCl}, \mathrm{CaCl}_{2}$ and their combinations 1:1 (w/w); and how to minimizing the harmful effects through pre-soaking seeds in vitamins (Ascorbic acid, a-tocopherol) or bio-regulator (Salicylic acid), or Yeast extract. The seeds of sweet pepper were sown on Jan, 13, 2008. A homogenous sweet pepper seeds were placed in $100 \mathrm{ml}$ beakers and $20 \mathrm{ml}$ of $1 \%$ sodium hypochlorite was added for sterilization. These were left in the solution for $5 \mathrm{~min}$ followed by washing under running tap water and ionized water twice. Then divided into 9 sets. The first set was soaked (24hours) in distilled water as control and the remaining sets (8) were separately soaked for $24 \mathrm{~h}$ in aqueous solution of AsA or $\alpha$-toco. at (50 or $100 \mathrm{mg} / \mathrm{L}$ ) each or SA at $(75$ or $150 \mathrm{mg} / \mathrm{L})$ or Yeast extract at (1000 or $2000 \mathrm{mg} / \mathrm{L}$ ). Then germinated in seedling trays (209 eye) containing peat moss and perlite (1:1) as a rooting medium moistured by nutrient cooper solution (Cooper, 1979). Trays containing the seeds were placed in a glasshouse at $28 \pm 2^{0} \mathrm{C}$ to germinate.

The experimental layout consisted of 7 automatic hydroponic units (groups) (experimental plots). Each hydroponic unit comprised of two plastic channels $(4 \mathrm{~m}$ long * $10 \mathrm{~cm}$ in diameter) placed on one side of the holder ( $4 \mathrm{~m}$ length * $1.5 \mathrm{~m}$ height). Each channel had 40 pores (6 cm diameter). Every unit was provided by an electric pump representing seven groups (Table, 1) nutrient solution $\left(2.0 \mathrm{dSm}^{-1}\right.$ as a control), $2000 \mathrm{mg} / \mathrm{L} \mathrm{NaCl}\left(5.42 \mathrm{dSm}^{-1}\right), 4000 \mathrm{mg} / \mathrm{L} \mathrm{NaCl}^{-1}\left(8.42 \mathrm{dSm}{ }^{-1}\right)$, $2000 \mathrm{mg} / \mathrm{L} \mathrm{CaCl}_{2}\left(4.59 \mathrm{dSm}^{-1}\right), 4000 \mathrm{mg} / \mathrm{L} \mathrm{CaCl}_{2}\left(7.60 \mathrm{dSm}^{-1}\right), 2000 \mathrm{mg} / \mathrm{L} \mathrm{NaCl}_{+} \mathrm{CaCl}_{2}(1: 1)\left(5.08 \mathrm{dSm}{ }^{-1}\right)$ and $4000 \mathrm{mg} / \mathrm{L}$ $\mathrm{NaCl}+\mathrm{CaCl}_{2}(1: 1)\left(8.08 \mathrm{dSm}^{-1}\right)$. 
Table (3): Composition of yeast extract (according to, Nagodawithana, 1991)

\begin{tabular}{|c|c|c|c|}
\hline \multicolumn{2}{|c|}{ Constituents } & \multicolumn{2}{|l|}{ Value (\%) } \\
\hline \multicolumn{2}{|l|}{ Protein } & \multicolumn{2}{|l|}{47} \\
\hline \multicolumn{2}{|c|}{ Carbohydrates } & \multicolumn{2}{|l|}{33} \\
\hline \multicolumn{2}{|l|}{ Minerals } & \multicolumn{2}{|l|}{8} \\
\hline \multicolumn{2}{|c|}{ Nucleic acids } & \multicolumn{2}{|l|}{8} \\
\hline \multicolumn{2}{|l|}{ Lipids } & \multicolumn{2}{|l|}{4} \\
\hline \multicolumn{4}{|c|}{ Approximate composition of vitamins } \\
\hline \multicolumn{2}{|l|}{ Vitamines } & \multicolumn{2}{|c|}{ Value $(\boldsymbol{\mu g} / \mathbf{g})$} \\
\hline \multicolumn{2}{|l|}{ Cholin } & \multicolumn{2}{|l|}{4000} \\
\hline \multicolumn{2}{|l|}{ Niacin } & \multicolumn{2}{|l|}{$300-500$} \\
\hline \multicolumn{2}{|c|}{ Thiamine $\left(\mathrm{B}_{1}\right)$} & \multicolumn{2}{|l|}{$60-100$} \\
\hline \multicolumn{2}{|c|}{ Pantorhenate $\left(\mathrm{B}_{5}\right)$} & \multicolumn{2}{|l|}{70} \\
\hline \multicolumn{2}{|c|}{ Riboflavin $\left(\mathrm{B}_{2}\right)$} & \multicolumn{2}{|l|}{$35-50$} \\
\hline \multicolumn{2}{|c|}{ Pyridoxine $\mathrm{HCL}\left(\mathrm{B}_{6}\right)$} & \multicolumn{2}{|l|}{28} \\
\hline \multicolumn{2}{|l|}{ Folic acid } & \multicolumn{2}{|l|}{$5-13$} \\
\hline \multicolumn{2}{|l|}{ Biotin } & \multicolumn{2}{|l|}{1.3} \\
\hline \multicolumn{2}{|l|}{ Vit. $B_{12}$} & \multicolumn{2}{|l|}{0.001} \\
\hline & Approxima & sition of $\mathrm{m}$ & \\
\hline Minerals & Value $(\mathrm{mg} / \mathrm{g})$ & Minerals & Value $(\mu g / g)$ \\
\hline $\mathrm{K}$ & 21 & $\mathrm{Cu}$ & 8.00 \\
\hline$P$ & 13.50 & $\mathrm{Ni}$ & 3.00 \\
\hline $\mathrm{S}$ & 3.90 & Sn & 3.00 \\
\hline $\mathrm{Mg}$ & 1.65 & $\mathrm{Cr}$ & 2.20 \\
\hline $\mathrm{Ca}$ & 0.75 & Mo & 0.40 \\
\hline $\mathrm{Zn}$ & 0.17 & $\mathrm{Se}$ & 0.10 \\
\hline $\mathrm{Na}$ & 0.12 & $\mathrm{Li}$ & 0.17 \\
\hline $\mathrm{Si}$ & 0.03 & $\mathrm{Va}$ & 0.04 \\
\hline $\mathrm{Fe}$ & 0.02 & $\mathrm{Mn}$ & 0.02 \\
\hline
\end{tabular}

The seedlings were transplanted to the experimental installation on Feb, 26 , 2008 (after 45 days from presoaking) at the stage of four/five true leaves. Two uniform seedlings were transplanted to $6 \mathrm{~cm}$ perforated pots (reticulated) containing peat moss and perlite (1:1) as a rooting medium.

Every two channels was divided into 9 sets, the first set was soaked in distilled water (control), AsA, $\alpha$-tocopherol at (50 or $100 \mathrm{mg} / \mathrm{L}$ ) each, SA at (75 or $150 \mathrm{mg} / \mathrm{L})$, and Yeast extract at (1000 or $2000 \mathrm{mg} / \mathrm{L})$. Each set contained (8 replicates) 16 seedlings (two seedling/pot) spaced $10 \mathrm{~cm}$ representing a Nutrient Film Technique (NFT).

To keep the concentrations of sodium chloride and mineral nutrients constant, the solution was changed every 7 to 10 days and the volume of the solution was maintained by adding distilled water as required after measuring the electrical conductivity by digital conductivity meter Lutron CD-4301. A nutrient solution was pumped into the channels at a flow rate of one liter per minute from a reservoir containing 10 liters.

\section{Sampling dates:}

Two fresh leaf samples were taken at 30 and 45 days after transplanting (75 and 90 days from sowing) to study the following measurements. 


\section{Phytochemical Constituents Analysis:}

Electrolyte leakagewas used to assess membrane permeability according to Lutts et al. (1996); Relative water content (RWC) was determined following the methods of (Jones and Turner, 1978); Photosynthetic pigments (mg/g FW) according to Mackiny (1941); ascorbic acid concentration according to (Sadasivam and Manickam, 1996), Proline concentration according to Troll and Lindsley (1955); Total soluble carbohydrates according to (Sadasivam and Manickam, 1996) and total phenols concentration according to (Kayani et al., 1990)

\section{Statistical analysis:}

The obtained data were subjected to statistical analysis of variance according to Gomez and Gomez (1984).

\section{RESULTS AND DISCUSSION}

\section{Electrolyte Leakage \%}

Data presented in Table (4) revealed that high salinity level of $\mathrm{NaCl}$ and $\mathrm{CaCl}_{2}(4000 \mathrm{mg} / \mathrm{L})$ markedly increased electrolyte leakage percentage (E.L. \%) in sweet pepper leaves. While, $\mathrm{NaCl}, \mathrm{CaCl}_{2}$ and its combinations at 2000 and $4000 \mathrm{mg} / \mathrm{L}$ caused a marked decrease in this respect as compared to the unstressed plants. In addition, application of all applied bio-stimulants at both applied levels decreased significantly E.L. \%. The most effective treatments were ascorbic acid at 50 and $100 \mathrm{mg} / \mathrm{L}$ at both sampling dates. As for the interaction between salinity type and concentration $\left(A^{*} B\right)$ it is clear that E.L. \% was decreased under low level of salinity $(2000 \mathrm{mg} / \mathrm{L})$. While, the maximum decrease was recorded under $\mathrm{NaCl}+\mathrm{CaCl}_{2}(1: 1)$ followed by $\mathrm{CaCl}_{2}$ and $\mathrm{NaCl}$, thereafter increased gradually with increasing salinity level. Meanwhile, under saline condition, application of the selected bio-stimulants, in most cases, counteracted the harmful effect of salinity on E.L. \%, especially, ascorbic acid at $50 \mathrm{mg} / \mathrm{L}$ as compared to untreated plants under saline conditions.

Membranes are the most important structural in plants for regulating ion content in the cells (Greenway and Munns, 1980). In addition, membrane structure and integrity are directly affected by changes in the ionic environment, where membrane damage representing as electrolyte leakage was increased with increasing salt concentration (Table, 4). Increasing the calcium/sodium ratio in the external solution alleviates the effect of salinity on depolarization and selectivity of the plasma membrane (Rinaldelli and Mancuso, 1996).

Moreover, the beneficial effects of calcium additions to the root environment of sodium chloride stressed plants are associated with the maintenance of cell membrane integrity, reducing sodium and favoring potassium absorption in salt stressed plants (Epstein, 1998).

\section{Relative water content (RWC)}

Data in Table (5) revealed that RWC in plant leaves grown under low levels of all of salinity types (2000 mg/L) $\mathrm{NaCl}, \mathrm{CaCl}_{2}$ and its combination (1:1) was increased significantly at 75 and 90 days from sowing. In addition, RWC \% under $\mathrm{CaCl}_{2}$ at $2000 \mathrm{mg} / \mathrm{L}$ showed a remarkable increase followed by $\mathrm{NaCl}+\mathrm{CaCl}_{2}(1: 1)$ and $\mathrm{NaCl}$ at $2000 \mathrm{mg} / \mathrm{L}$. On the other hand, increasing salinity from 2000 to $4000 \mathrm{mg} / \mathrm{L}$ decreased gradually RWC and gave the great reduction at the highest salinity level of $\mathrm{NaCl}$.

However, pre-soaking seeds in selected chemicals used (AsA, a-tocopherol SA, or yeast) at both levels had a significant increase on RWC \% under non-saline condition. Furthermore, pepper seeds presoaked in AsA at $50 \mathrm{mg} / \mathrm{L}$, SA at $75 \mathrm{mg} / \mathrm{L}$ or $\alpha$-tocopherol at $50 \mathrm{mg} / \mathrm{L}$ resulted in higher RWC \% as compared with the other treatments.

Regarding the interactions, between salinity levels and the applied bio-stimulants $\left(A^{*} \mathrm{C}\right)$ the data in the same table indicated that RWC \% was significantly increased as compared with unstressed seeds. It inferred from the results obtained in this experiment that all treatments enhanced RWC \%under high salinity levels. Furthermore, AsA at $50 \mathrm{mg} / \mathrm{L}$ or SA at $75 \mathrm{mg} / \mathrm{L}$ was more effective in this respect as compared to the untreated plants grown under non-saline or saline conditions. The decrease in RWC\% could be attributed to root systems which are not able to compensate water lost by transpiration through a reduction of the absorbing surface (Gadallah, 2000). 
Table (4): Effect of pre-soaking seeds in SA, AsA, $\alpha$-tocopherol or Yeast extract on Electrolyte Leakage \% of sweet pepper grown under non-saline and saline conditions at 75 and 90 days from sowing using NFT.

\begin{tabular}{|c|c|c|c|c|c|c|c|c|c|c|c|c|}
\hline \multirow{3}{*}{\multicolumn{2}{|c|}{$\begin{array}{l}\text { Salinity } \\
\quad(A) \\
\text { Treatment } \\
\text { (C) } \mathrm{mg} / \mathrm{L}\end{array}$}} & \multirow[b]{3}{*}{ N.S. } & \multicolumn{3}{|c|}{ N.S.+ $\mathrm{NaCl}$} & \multicolumn{3}{|c|}{ N.S. $+\mathrm{CaCl}_{2}$} & \multicolumn{3}{|c|}{$\begin{array}{c}\text { N.S. }+\left(\mathrm{NaCl}+\mathrm{CaCl}_{2}\right) \\
(1: 1) \mathrm{w} / \mathrm{w}\end{array}$} & \multirow{3}{*}{$\begin{array}{l}\text { Mean } \\
(\mathrm{C})\end{array}$} \\
\hline & & & \multicolumn{2}{|c|}{ Conc. (B) } & \multirow{2}{*}{$\begin{array}{l}\text { Mean } \\
\left(A^{*} C\right)\end{array}$} & \multicolumn{2}{|c|}{ Conc. (B) } & \multirow{2}{*}{$\begin{array}{l}\text { Mean } \\
\left(A^{*} C\right)\end{array}$} & \multicolumn{2}{|c|}{ Conc. (B) } & \multirow{2}{*}{$\begin{array}{l}\text { Mean } \\
\left(A^{*} C\right)\end{array}$} & \\
\hline & & & 2000 & 4000 & & 2000 & 4000 & & 2000 & 4000 & & \\
\hline \multicolumn{13}{|c|}{75 days from sowing } \\
\hline \multicolumn{2}{|c|}{ Water } & 31.57 & 30.13 & 42.60 & 34.77 & 29.10 & 32.50 & 31.06 & 25.80 & 28.97 & 28.78 & 31.53 \\
\hline \multicolumn{2}{|c|}{ SA 75} & 29.10 & 26.63 & 38.87 & 31.53 & 27.73 & 24.70 & 27.18 & 24.17 & 28.07 & 27.11 & 28.61 \\
\hline \multicolumn{2}{|c|}{ SA 150} & 24.90 & 27.60 & 38.13 & 30.21 & 25.43 & 29.07 & 26.47 & 21.03 & 28.27 & 24.73 & 27.14 \\
\hline \multicolumn{2}{|c|}{ AsA 50} & 28.57 & 29.13 & 38.57 & 32.09 & 27.63 & 28.33 & 28.18 & 19.53 & 25.93 & 24.68 & 28.31 \\
\hline \multicolumn{2}{|c|}{ AsA 100} & 25.07 & 25.57 & 41.10 & 30.58 & 23.63 & 26.53 & 25.08 & 18.33 & 21.40 & 21.60 & 25.75 \\
\hline \multicolumn{2}{|c|}{ a-toco 50} & 24.77 & 27.10 & 37.40 & 29.76 & 25.20 & 31.60 & 27.19 & 19.40 & 22.60 & 22.26 & 26.40 \\
\hline \multicolumn{2}{|c|}{ a-toco 100} & 24.93 & 22.20 & 35.87 & 27.67 & 21.43 & 28.53 & 24.97 & 23.80 & 25.43 & 24.72 & 25.79 \\
\hline Yea & 1000 & 30.47 & 30.33 & 35.10 & 31.97 & 26.60 & 30.47 & 29.18 & 23.40 & 23.83 & 25.90 & 29.01 \\
\hline Yea & 2000 & 29.70 & 27.83 & 35.13 & 30.89 & 26.13 & 28.63 & 28.16 & 23.50 & 21.70 & 24.97 & 28.00 \\
\hline & $A$ & & 31.05 & & & 27.49 & & & 24.97 & & & \\
\hline త్ర్ల & B & 27.67 & 25.13 & 30.72 & & & & & & & & \\
\hline 2 & $A^{*} B$ & & 27.39 & 38.09 & & 25.88 & 28.93 & & 22.11 & 25.13 & & \\
\hline LSL & 0.05 & $\mathrm{~A} ; 0.44$ & $\mathrm{~B} ; 0$ & 44 & C; 0.75 & $A^{*} B ; 0$. & & $A^{*} C ; 1.3$ & $\mathrm{~B}^{*} \mathrm{C}$ & 1.31 & $A^{*} B^{\star} C$ & ; 2.26 \\
\hline & & & & & $90 \mathrm{da}$ & 8 & sowing & & & & & \\
\hline $\mathrm{Wa}$ & & 51.00 & 46.27 & 62.73 & 53.33 & 44.97 & 55.07 & 50.34 & 35.83 & 45.67 & 44.17 & 49.28 \\
\hline SA & & 42.50 & 47.10 & 54.53 & 48.04 & 35.50 & 51.67 & 43.22 & 32.27 & 43.17 & 39.31 & 43.53 \\
\hline SA & & 45.80 & 40.77 & 54.60 & 47.06 & 33.97 & 52.50 & 44.09 & 34.03 & 45.43 & 41.76 & 44.30 \\
\hline Ast & & 39.63 & 42.17 & 50.77 & 44.19 & 30.10 & 51.00 & 40.24 & 33.07 & 49.43 & 40.71 & 41.71 \\
\hline Ast & & 51.20 & 51.90 & 52.57 & 51.89 & 25.83 & 52.20 & 43.08 & 27.10 & 34.60 & 37.63 & 44.20 \\
\hline$\alpha-t c$ & 50 & 43.60 & 50.40 & 56.33 & 50.11 & 25.37 & 38.67 & 35.88 & 34.83 & 49.53 & 42.66 & 42.88 \\
\hline$\alpha-t c$ & 100 & 43.23 & 50.07 & 55.13 & 49.48 & 32.07 & 47.33 & 40.88 & 29.27 & 45.33 & 39.28 & 43.21 \\
\hline Yea & 1000 & 40.57 & 44.80 & 54.10 & 46.49 & 32.53 & 50.97 & 41.36 & 31.53 & 39.03 & 37.04 & 41.63 \\
\hline Yea & 2000 & 46.63 & 48.83 & 55.67 & 50.38 & 29.30 & 52.80 & 42.91 & 52.73 & 33.67 & 44.34 & 45.88 \\
\hline & $A$ & & 49.00 & & & 42.44 & & & & 40. & & \\
\hline 胥 & $B$ & 44.91 & 37.87 & 49.43 & & & & & & & & \\
\hline & $A^{*} B$ & & 46.92 & 55.16 & & 32.18 & 50.24 & & 34.52 & 42.87 & & \\
\hline$\overline{\mathrm{LSL}}$ & 0.05 & $A ; 0.89$ & $\mathrm{~B} ; 0$ & & C; 1.54 & $A^{*} B ; 1$ & & $A^{*} C ; 2.6$ & $\mathrm{~B}^{*} \mathrm{C}$ & 2.66 & $A^{*} B$ & 4.62 \\
\hline
\end{tabular}

\begin{tabular}{|c|c|}
\hline N.S. = Nutrient Solution (Control) & $\mathrm{SA}=$ Salicylic acid \\
\hline $\mathrm{A} s \bar{A}=$ Ascorbic acid & $\alpha$-toco. = a-tocopherol \\
\hline
\end{tabular}


Table (5): Effect of pre-soaking seeds in SA, AsA, a-tocopherol or Yeast extract on Relative water content \% of sweet pepper grown under non-saline and saline conditions at 75 and 90 days from sowing using NFT.

\begin{tabular}{|c|c|c|c|c|c|c|c|c|c|c|c|c|}
\hline \multirow{3}{*}{\multicolumn{2}{|c|}{$\begin{array}{c}\text { Salinity } \\
\text { (A) } \\
\text { Treatment }(C) \\
\text { mg/L }\end{array}$}} & \multirow[b]{3}{*}{ N.S. } & \multicolumn{3}{|c|}{ N.S. $+\mathrm{NaCl}$} & \multicolumn{3}{|c|}{ N.S.+ $\mathrm{CaCl}_{2}$} & \multicolumn{3}{|c|}{$\begin{array}{c}\text { N.S. }+\left(\mathrm{NaCl}+\mathrm{CaCl}_{2}\right) \\
(1: 1) \mathrm{w} / \mathrm{w}\end{array}$} & \multirow{3}{*}{$\begin{array}{l}\text { Mean } \\
\text { (C) }\end{array}$} \\
\hline & & & \multicolumn{2}{|c|}{ Conc. (B) } & \multirow{2}{*}{$\begin{array}{l}\text { Mean } \\
\left(A^{*} C\right)\end{array}$} & \multicolumn{2}{|c|}{ Conc. (B) } & \multirow{2}{*}{$\begin{array}{l}\text { Mean } \\
\left(A^{*} C\right)\end{array}$} & \multicolumn{2}{|c|}{ Conc. (B) } & \multirow{2}{*}{$\begin{array}{l}\text { Mean } \\
\left(A^{*} C\right)\end{array}$} & \\
\hline & & & 2000 & 4000 & & 2000 & 4000 & & 2000 & 4000 & & \\
\hline \multicolumn{13}{|c|}{75 days from sowing } \\
\hline \multicolumn{2}{|c|}{ Water } & 42.88 & 50.38 & 41.56 & 42.88 & 76.48 & 46.76 & 42.88 & 69.28 & 48.88 & 51.33 & 42.88 \\
\hline \multicolumn{2}{|c|}{ SA 75} & 54.58 & 62.47 & 43.86 & 54.58 & 80.75 & 53.98 & 54.58 & 72.04 & 42.86 & 57.74 & 54.58 \\
\hline \multicolumn{2}{|c|}{ SA 150} & 57.97 & 59.26 & 40.35 & 57.97 & 79.73 & 60.23 & 57.97 & 70.52 & 53.65 & 59.74 & 57.97 \\
\hline \multicolumn{2}{|c|}{ AsA 50} & 53.87 & 60.34 & 51.26 & 53.87 & 71.51 & 58.42 & 53.87 & 68.82 & 46.11 & 57.56 & 53.87 \\
\hline \multicolumn{2}{|c|}{ AsA 100} & 51.30 & 65.92 & 49.39 & 51.30 & 72.56 & 57.60 & 51.30 & 61.96 & 50.83 & 56.91 & 51.30 \\
\hline \multicolumn{2}{|c|}{ a-toco 50} & 65.64 & 64.45 & 62.45 & 65.64 & 78.38 & 50.08 & 65.64 & 68.07 & 59.16 & 64.39 & 65.64 \\
\hline \multicolumn{2}{|c|}{ a-toco 100} & 57.31 & 71.69 & 54.15 & 57.31 & 80.77 & 52.24 & 57.31 & 71.59 & 54.23 & 61.84 & 57.31 \\
\hline \multicolumn{2}{|c|}{ Yeast 1000} & 46.13 & 57.45 & 44.09 & 46.13 & 78.51 & 50.01 & 46.13 & 65.22 & 54.28 & 54.22 & 46.13 \\
\hline \multicolumn{2}{|c|}{ Yeast 2000} & 51.34 & 52.80 & 39.60 & 51.34 & 64.84 & 48.09 & 51.34 & 81.67 & 53.06 & 54.90 & 51.34 \\
\hline \multirow{3}{*}{$\begin{array}{l}\stackrel{ }{\mathbb{N}} \\
\stackrel{\mathbb{N}}{\Sigma}\end{array}$} & & \multirow{3}{*}{53.45} & & 53.80 & & & 60.81 & & & 58.27 & & \\
\hline & & & 68.79 & 50.64 & & & & & & & & \\
\hline & $A^{*} B$ & & 60.53 & 47.41 & & 75.95 & 53.05 & & 69.91 & 51.45 & & \\
\hline \multicolumn{2}{|c|}{ LSD at 0.05} & $A ; 2.0$ & \multicolumn{2}{|c|}{$\mathrm{B} ; 2.01$} & C; 3.48 & $A^{*} B$ & 3.48 & $A^{*} C ; 4.26$ & $\mathrm{~B}^{*} \mathrm{C}$ & ; 4.26 & $A^{*} B^{*} C$ & 10.44 \\
\hline & & & & & $90 \mathrm{c}$ & ys 1100 & sowing & & & & & \\
\hline & ater & 62.32 & 64.40 & 48.62 & 58.45 & 80.78 & 51.79 & 64.96 & 67.66 & 49.37 & 59.78 & 61.06 \\
\hline & 75 & 72.41 & 85.63 & 61.96 & 73.33 & 90.38 & 74.75 & 79.18 & 90.79 & 67.63 & 76.94 & 76.49 \\
\hline & 150 & 67.75 & 75.96 & 61.49 & 68.40 & 83.12 & 66.60 & 72.49 & 78.77 & 62.64 & 69.72 & 70.20 \\
\hline & A 50 & 77.62 & 85.36 & 66.35 & 76.44 & 91.35 & 72.25 & 80.41 & 86.11 & 64.57 & 76.10 & 77.65 \\
\hline & 100 & 69.03 & 72.61 & 55.46 & 65.70 & 91.26 & 66.51 & 75.60 & 77.45 & 60.84 & 69.11 & 70.14 \\
\hline & co 50 & 77.02 & 78.93 & 55.94 & 70.63 & 86.67 & 57.44 & 73.71 & 78.37 & 56.29 & 70.56 & 71.63 \\
\hline & 20100 & 63.83 & 78.26 & 56.77 & 66.29 & 95.63 & 73.57 & 77.68 & 83.70 & 62.59 & 70.04 & 71.33 \\
\hline & 1000 & 62.71 & 71.87 & 52.27 & 62.29 & 87.91 & 58.16 & 69.59 & 72.65 & 52.60 & 62.65 & 64.84 \\
\hline & 2000 & 67.28 & 77.36 & 53.10 & 65.92 & 89.95 & 59.60 & 72.28 & 82.37 & 59.76 & 69.80 & 69.33 \\
\hline & & & & 67.49 & & & 73.99 & & & 69.41 & & \\
\hline$\sum^{\infty}$ & & 68.89 & 81.68 & 60.33 & & & & & & & & \\
\hline & $A^{*} B$ & & 76.71 & 56.88 & & 88.56 & 64.52 & & 79.76 & 59.59 & & \\
\hline & 0.05 & $A ; 1.9$ & & 1.94 & $\mathrm{C} ; 3.36$ & $A^{*} B$ & 3.36 & $A^{*} C ; 5.82$ & & ; 5.82 & $A^{*} B$ & 10.08 \\
\hline
\end{tabular}

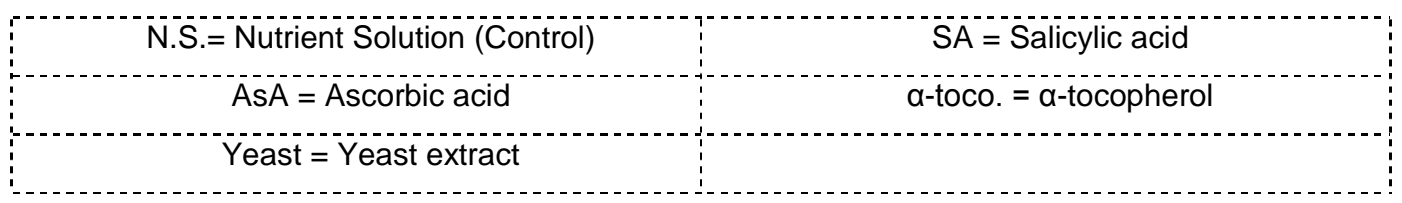




\section{Photosynthetic pigments concentration}

The data in Tables (6-8) clearly show that low salinity levels of all salinity types $\mathrm{NaCl}, \mathrm{CaCl}_{2}$ and its combination $(1: 1 \mathrm{w} / \mathrm{w})$ at $2000 \mathrm{mg} / \mathrm{L}$ caused a high significant increase in the photosynthetic pigments concentrations in sweet pepper leaves (total chlorophylls, carotenoids, chlorophyll $\mathrm{a}, \mathrm{b}$ as well as chlorophyll a/b ratio) while chlorophyll/carotenoids ratio was decreased after 90 days from sowing. Moreover, sweet pepper plants growing under $\mathrm{NaCl}+\mathrm{CaCl} 2(1: 1)$ at $2000 \mathrm{mg} / \mathrm{L}$ resulted a greater increase in total chlorophylls, carotenoids, chlorophyll $a, b$ as well as chlorophyll $a / b$ ratio, and decreased chlorophyll/carotenoids ratio followed by $\mathrm{CaCl} 2$ and $\mathrm{NaCl}$ at the same level. In addition, increasing salinity levels from 2000 to $4000 \mathrm{mg} / \mathrm{L}$ caused a significant decrease in the photosynthetic pigments concentrations.

As regard to the effects of pre-soaking seeds in SA, AsA, $\alpha$-tocopherol and yeast extract at both levels, total chlorophylls, carotenoids, chlorophyll $\mathrm{a}, \mathrm{b}$ and chlorophyll $\mathrm{a} / \mathrm{b}$ ratio as well as chlorophyll/carotenoids ratio were increased under nonsaline conditions. In addition, pre-soaking seeds in SA at $75 \mathrm{mg} / \mathrm{L}$ was more effective as compared with the other treatments. Moreover, means $\left(A^{*} B\right)$ indicated that total chlorophyll, carotenoids, chl. a concentrations as well as chlorophyll a/b ratio were significantly increased under low salinity level of all salinity types at $2000 \mathrm{mg} / \mathrm{L}$.

From the above mentioned results it could be concluded that photosynthetic pigments (chlorophyll $a, b$ and their total as well as carotenoids) concentrations in the leaves of sweet pepper were increased under low salinity level of $\mathrm{NaCl}$, $\mathrm{CaCl} 2$ as well as its combination, thereafter decreased with increasing salinity level as presented in Tables(6-8) and also supported by El-Banna (2006) and Arafa et al. (2009). Moreover, all applied bio-stimulants enhanced photosynthetic pigments concentrations under high salinity levels $(4000 \mathrm{mg} / \mathrm{L})$ and $\mathrm{SA}$ at $75 \mathrm{mg} / \mathrm{L}$ was more effective in this respect.

The stimulating effect of low salinity level $\left(\mathrm{NaCl}, \mathrm{CaCl}_{2}\right.$ as well as its combination) on photosynthetic pigments may be due to enhancing cytokinin, auxin and GAs content, which stimulated chlorophyll and delay chlorophyll destruction then delay senescence (Ghallab and Nesiem, 1999).

The reduction in chlorophylls was accompanied with an irregular fluctuation values regarding chl a/b ratio whereas, chlorophylls/carotenoids ratio decreased due to the increase in carotenoids values and the decrease in chlorophylls. These reduction in photosynthetic pigments was related to an enhancement in the activity of chlorophyll degradation enzyme chlorophyllase (Saha et al., 2010) and/or the inhibitions effects of chloride on the activity Fe-containing enzymes cytochrome oxidase, which may decrease the rate of chlorophyll biosynthesis as well as an increase in chlorophyll degradation (Santos et al., 2001) and increased ABA content resulting in promoting chlorophyll breakdown and leaf senescence (Hatung, 2004), and/or a disturbed chloroplast structure, number and size which affected chlorophyll content (Arafa et al., 2009) In the present study, salinity decreased carotenoid content (Table, 6) due to degradation of $\alpha$-carotein and formation of zeaxanthin, which protect the plant against photoinhibition (Sharma and Hall, 1991).

The stimulating effect of phytohoromnes, vitamins and yeast extract on photosynthetic pigments concentration may be due to; stabilizing active site of enzymes (Hare et al., 1998). Furthermore, SA caused considerable enhancement in photosynthetic pigments under non-saline or salt stress which may be due to increased auxin and zeatin as well as gibberellin in leaves (Shehata et al., 2000) and/or increased the rate of photosynthetic electron transport and/or increased the photochemical quenching parameter in the presence of sodium (Tari et al., 2002) and/or activated the synthesis of carotenoids and decreased chlorophyll pigments (Moharekar et al., 2003).

Concerning AsA, it caused considerable enhancement in photosynthetic pigments under non-saline and salt stress which may be due to the fact that it is an important primary metabolite in plants that functions as an antioxidant, an enzyme cofactor and a cell signalling modulator in a wide array of crucial physiological processes, including biosynthesis of the cell wall, secondary metabolites and phytohoromnes, stress tolerance, photoprotection, cell division and growth (Wolucka et al., 2005) 
Table (6): Effect of pre-soaking seeds in SA, AsA, a-tocopherol or Yeast extract on total chlorophylls and carotenoids concentrations $(\mathrm{mg} / \mathrm{g} \mathrm{FW})$ in sweet pepper leaves under non-saline and saline conditions at 75 and 90 days from sowing using NFT.

\begin{tabular}{|c|c|c|c|c|c|c|c|c|c|c|c|c|}
\hline \multirow{3}{*}{\multicolumn{2}{|c|}{$\begin{array}{c}\text { Salinity } \\
\text { (A) } \\
\text { Treatment }(C) \\
\text { mg/L }\end{array}$}} & \multirow[b]{3}{*}{ N.S. } & \multicolumn{3}{|c|}{ N.S.+ NaCl } & \multicolumn{3}{|c|}{ N.S. $+\mathrm{CaCl}_{2}$} & \multicolumn{3}{|c|}{$\begin{array}{c}\text { N.S.+ }\left(\mathrm{NaCl}+\mathrm{CaCl}_{2}\right) \\
(1: 1) \mathrm{w} / \mathrm{w}\end{array}$} & \multirow{3}{*}{$\begin{array}{l}\text { Mean } \\
\text { (C) }\end{array}$} \\
\hline & & & \multicolumn{2}{|c|}{ Conc. (B) } & \multirow{2}{*}{$\begin{array}{l}\text { Mean } \\
\left(A^{*} C\right)\end{array}$} & \multicolumn{2}{|c|}{ Conc. (B) } & \multirow{2}{*}{$\begin{array}{l}\text { Mean } \\
\left(A^{*} C\right)\end{array}$} & \multicolumn{2}{|c|}{ Conc. (B) } & \multirow{2}{*}{$\begin{array}{l}\text { Mean } \\
\left(A^{*} C\right)\end{array}$} & \\
\hline & & & 2000 & 4000 & & 2000 & 4000 & & 2000 & 4000 & & \\
\hline \multicolumn{13}{|c|}{ Total chlorophylls } \\
\hline \multicolumn{2}{|c|}{ Water } & 1.47 & 1.56 & 0.89 & 1.31 & 1.64 & 1.42 & 1.51 & $\begin{array}{ll}1.73 \\
\end{array}$ & 1.30 & 1.50 & 1.44 \\
\hline \multicolumn{2}{|c|}{ SA 75} & 1.55 & 1.62 & 1.30 & 1.49 & 1.71 & 1.47 & 1.57 & 2.21 & 1.42 & 1.72 & 1.60 \\
\hline \multicolumn{2}{|c|}{ SA 150} & 1.53 & 1.59 & 1.18 & 1.43 & 1.66 & 1.45 & 1.55 & 1.91 & 1.37 & 1.60 & 1.53 \\
\hline \multicolumn{2}{|c|}{ AsA 50} & 1.54 & 1.61 & 1.24 & 1.46 & 1.70 & 1.47 & 1.57 & 2.19 & 1.42 & 1.72 & 1.58 \\
\hline \multicolumn{2}{|c|}{ AsA 100} & 1.54 & 1.61 & 1.20 & 1.45 & 1.68 & 1.46 & 1.56 & 1.95 & 1.40 & 1.63 & 1.55 \\
\hline \multicolumn{2}{|c|}{$\alpha$-toco 50} & 1.54 & 1.61 & 1.19 & 1.45 & 1.68 & 1.46 & 1.56 & 1.92 & 1.39 & 1.62 & 1.54 \\
\hline \multicolumn{2}{|c|}{ a-toco 100} & 1.54 & 1.61 & 1.21 & 1.45 & 1.69 & 1.46 & 1.56 & 1.99 & 1.41 & 1.65 & 1.55 \\
\hline \multicolumn{2}{|c|}{ Yeast 1000} & 1.49 & 1.56 & 1.15 & 1.40 & 1.64 & 1.43 & 1.52 & 1.79 & 1.32 & 1.53 & 1.49 \\
\hline \multicolumn{2}{|c|}{ Yeast 2000} & 1.52 & 1.57 & 1.14 & 1.41 & 1.65 & 1.44 & 1.54 & 1.83 & 1.34 & 1.56 & 1.50 \\
\hline \multirow{3}{*}{ 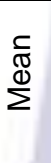 } & & \multirow{3}{*}{1.53} & \multicolumn{3}{|c|}{1.43} & \multicolumn{3}{|c|}{1.55} & \multicolumn{3}{|c|}{1.62} & \\
\hline & $B$ & & 1.74 & 1.33 & & & & & & & & \\
\hline & $A^{*} B$ & & 1.59 & 1.17 & 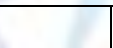 & 1.67 & 1.45 & & 1.95 & 1.38 & & \\
\hline & 0.05 & $A ; 0.0$ & $\mathrm{~B}$; & 0.04 & C; 0.06 & $A^{*} B$ & .06 & $A^{*} C ; 0.10$ & $\mathrm{~B}^{*}$ & 0.10 & $A^{*} B^{*}$ & $; 0.18$ \\
\hline & & & & & & & ioids & & & & & \\
\hline & ater & 0.610 & 0.673 & 0.490 & 0.591 & 0.753 & 0.573 & 0.646 & 0.877 & 0.540 & 0.676 & 0.637 \\
\hline & 75 & 0.663 & 0.740 & 0.530 & 0.644 & 0.847 & 0.610 & 0.707 & 1.110 & 0.573 & 0.782 & 0.711 \\
\hline & 150 & 0.657 & 0.730 & 0.517 & 0.634 & 0.790 & 0.593 & 0.680 & 1.003 & 0.560 & 0.740 & 0.685 \\
\hline & 450 & 0.660 & 0.740 & 0.523 & 0.641 & 0.827 & 0.607 & 0.698 & 1.107 & 0.570 & 0.779 & 0.706 \\
\hline & 100 & 0.660 & 0.737 & 0.517 & 0.638 & 0.797 & 0.600 & 0.686 & 1.040 & 0.570 & 0.757 & 0.693 \\
\hline & 50 & 0.657 & 0.723 & 0.500 & 0.627 & 0.790 & 0.600 & 0.682 & 1.020 & 0.567 & 0.748 & 0.686 \\
\hline & 0100 & 0.660 & 0.740 & 0.523 & 0.641 & 0.827 & 0.603 & 0.697 & 1.057 & 0.570 & 0.762 & 0.700 \\
\hline & 1000 & 0.620 & 0.687 & 0.487 & 0.598 & 0.753 & 0.577 & 0.650 & 0.890 & 0.547 & 0.686 & 0.644 \\
\hline & 2000 & 0.640 & 0.707 & 0.497 & 0.614 & 0.777 & 0.587 & 0.668 & 0.957 & 0.550 & 0.716 & 0.666 \\
\hline & & & & 0.625 & & & 0.679 & & & 0.738 & & \\
\hline 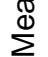 & & 0.647 & 0.841 & 0.555 & & & & & & & & \\
\hline & $A^{*} B$ & & 0.720 & 0.509 & & 0.796 & 0.594 & & 1.007 & 0.561 & & \\
\hline & 0.05 & $A ; 0.02$ & $\mathrm{~B}$; & .023 & ; 0.041 & $A^{*} B$ & .041 & $A^{*} C ; 0.07$ & $\mathrm{~B}^{*} \mathrm{C}$ & $; 0.070$ & $A^{*} B$ & 0.120 \\
\hline
\end{tabular}

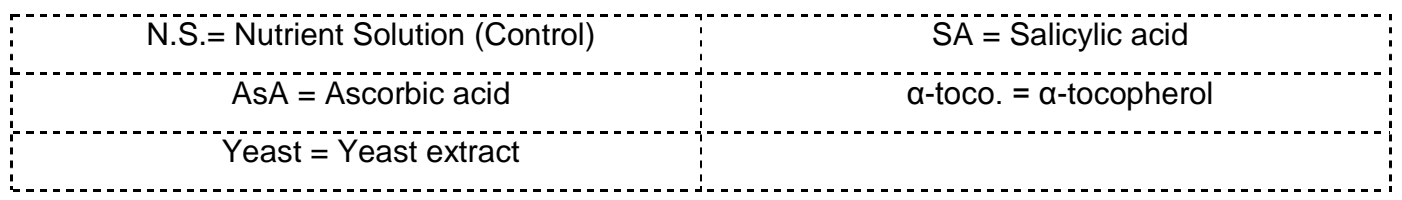


Table (7): Effect of pre-soaking seeds in SA, AsA, $\alpha$-tocopherol or Yeast extract on chlorophyll a and b concentrations $(\mathrm{mg} / \mathrm{g} \mathrm{FW})$ in sweet pepper leaves under non-saline and saline conditions at 75 and 90 days from sowing using NFT.

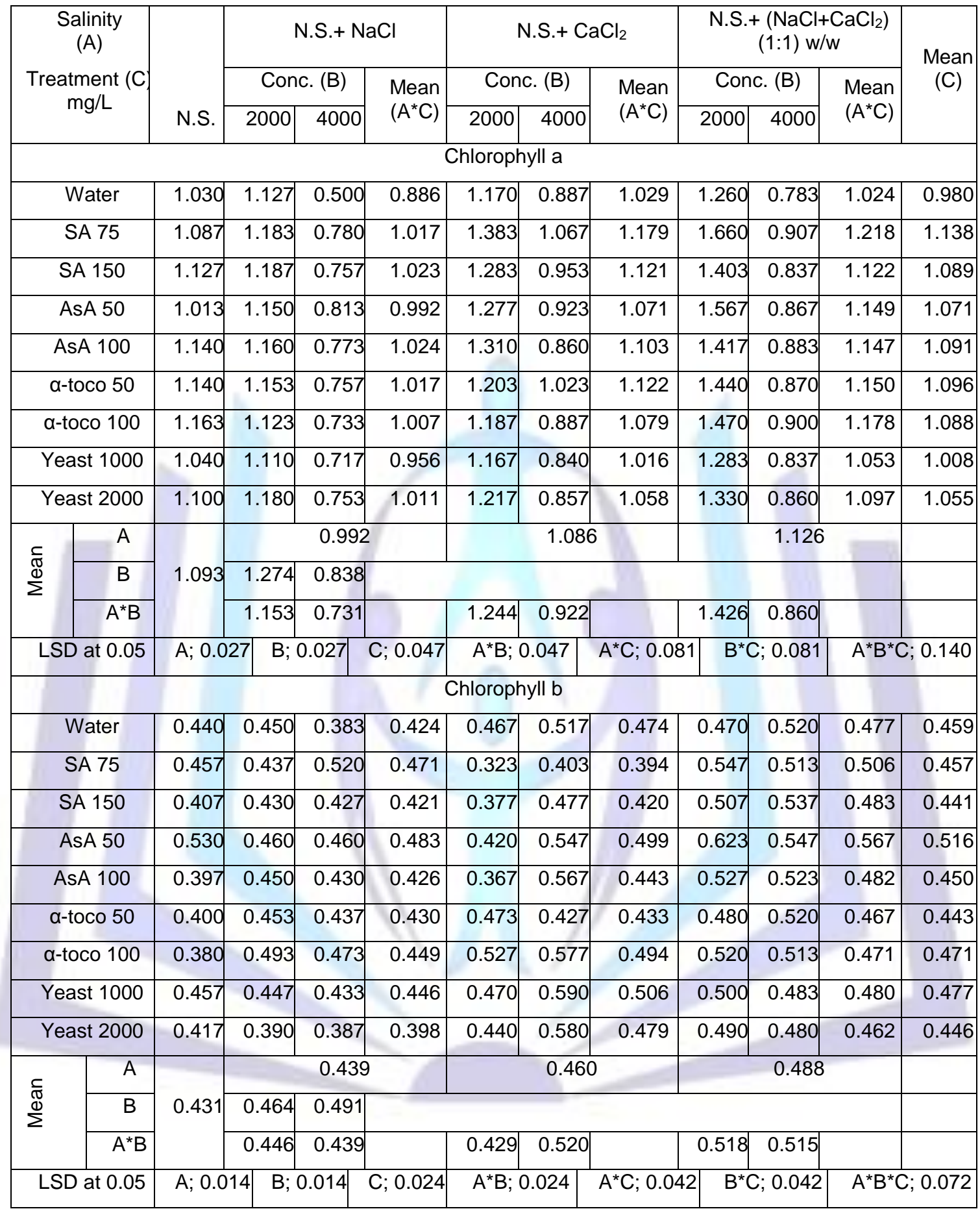

\begin{tabular}{|c|c|}
\hline N.S. $=$ Nutrient Solution (Control) & $\mathrm{SA}=$ Salicylic acid \\
\hline AsA $=$ Ascorbic acid & $\alpha$-toco. $=\alpha$-tocopherol \\
\hline
\end{tabular}


Table (8): Effect of pre-soaking seeds in SA, AsA, a-tocopherol or Yeast extract on chlorophyll a/b ratio and total chlorophylls/carotenoids ratio in sweet pepper leaves under non-saline and saline conditions at 75 and 90 days from sowing using NFT.

\begin{tabular}{|c|c|c|c|c|c|c|c|c|c|c|c|c|}
\hline \multirow{3}{*}{\multicolumn{2}{|c|}{$\begin{array}{c}\text { Salinity } \\
\text { (A) } \\
\text { Treatment }(\mathrm{C} \\
\mathrm{mg} / \mathrm{L}\end{array}$}} & \multirow[b]{3}{*}{ N.S. } & \multicolumn{3}{|c|}{ N.S. $+\mathrm{NaCl}$} & \multicolumn{3}{|c|}{ N.S. $+\mathrm{CaCl}_{2}$} & \multicolumn{3}{|c|}{$\begin{array}{c}\text { N.S. }+\left(\mathrm{NaCl}+\mathrm{CaCl}_{2}\right) \\
(1: 1) \mathrm{w} / \mathrm{w}\end{array}$} & \multirow{3}{*}{$\begin{array}{l}\text { Mean } \\
(\mathrm{C})\end{array}$} \\
\hline & & & \multicolumn{2}{|c|}{ Conc. (B) } & \multirow{2}{*}{$\begin{array}{l}\text { Mean } \\
\left(A^{*} C\right)\end{array}$} & \multicolumn{2}{|c|}{ Conc. (B) } & \multirow{2}{*}{$\begin{array}{l}\text { Mean } \\
\left(A^{*} C\right)\end{array}$} & \multicolumn{2}{|c|}{ Conc. (B) } & \multirow{2}{*}{$\begin{array}{c}\text { Mean } \\
\left(A^{*} C\right)\end{array}$} & \\
\hline & & & 2000 & 4000 & & 2000 & 4000 & & 2000 & 4000 & & \\
\hline \multicolumn{13}{|c|}{ Chlorophyll a/b ratio } \\
\hline \multicolumn{2}{|c|}{ Water } & 2.36 & 2.51 & 1.32 & 2.06 & 2.51 & 1.73 & 2.20 & 2.68 & 1.52 & 2.19 & 2.15 \\
\hline \multicolumn{2}{|c|}{ SA 75} & 2.38 & 2.71 & 1.50 & 2.20 & 4.26 & 2.65 & 3.10 & 3.17 & 1.77 & 2.44 & 2.58 \\
\hline \multicolumn{2}{|c|}{ SA 150} & 2.77 & 2.79 & 1.77 & 2.44 & 3.42 & 1.99 & 2.72 & 2.76 & 1.53 & 2.35 & 2.51 \\
\hline \multicolumn{2}{|c|}{ AsA 50} & 1.92 & 2.49 & 1.75 & 2.05 & 3.07 & 1.69 & 2.22 & 2.51 & 1.57 & 2.00 & 2.09 \\
\hline \multicolumn{2}{|c|}{ AsA 100} & 2.89 & 2.58 & 1.80 & 2.42 & 3.57 & 1.53 & 2.66 & 2.69 & 1.70 & 2.43 & 2.50 \\
\hline \multicolumn{2}{|c|}{ a-toco 50} & 2.86 & 2.55 & 1.73 & 2.38 & 2.54 & 2.39 & 2.60 & 3.03 & 1.67 & 2.52 & 2.50 \\
\hline \multicolumn{2}{|c|}{ a-toco 100} & 3.16 & 2.28 & 1.56 & 2.33 & 2.26 & 1.54 & 2.32 & 2.86 & 1.75 & 2.59 & 2.42 \\
\hline \multicolumn{2}{|c|}{ Yeast 1000} & 2.28 & 2.50 & 1.66 & 2.15 & 2.46 & 1.43 & 2.06 & 2.55 & 1.73 & 2.19 & 2.13 \\
\hline \multicolumn{2}{|c|}{ Yeast 2000} & 2.63 & 3.08 & 1.95 & 2.55 & 2.79 & 1.49 & 2.31 & 2.71 & 1.79 & 2.38 & 2.41 \\
\hline \multirow{3}{*}{ 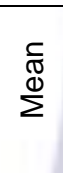 } & $A$ & \multirow{3}{*}{2.58} & \multicolumn{3}{|c|}{2.29} & \multicolumn{3}{|c|}{2.47} & \multicolumn{3}{|c|}{2.34} & \\
\hline & & & 2.79 & 1.72 & & & & & & & & \\
\hline & $A^{*} B$ & & 2.61 & 1.67 & & 2.99 & 1.83 & & 2.77 & 1.67 & & \\
\hline & 0.05 & $A ; 0.0$ & $\mathrm{~B}$; & 0.08 & $\mathrm{C} ; 0.14$ & $A^{*} B$ & & $\mathrm{~A}^{*} \mathrm{C} ; 0.24$ & & $; 0.24$ & $A^{*} B^{*}$ & $; 0.41$ \\
\hline & & & & & Chlorop & III/ Cal & teritusa & ratio & & & & \\
\hline & ter & 2.40 & 2.32 & 1.82 & 2.18 & 2.20 & 2.49 & 2.36 & 1.98 & 2.42 & 2.27 & 2.27 \\
\hline & 75 & 2.34 & 2.20 & 2.49 & 2.34 & 2.03 & 2.46 & 2.28 & 2.07 & 2.51 & 2.31 & 2.31 \\
\hline & 150 & 2.35 & 2.16 & 2.30 & 2.27 & 2.12 & 2.47 & 2.31 & 1.90 & 2.43 & 2.23 & 2.27 \\
\hline & 50 & 2.33 & 2.22 & 2.50 & 2.35 & 2.11 & 2.47 & 2.30 & 1.98 & 2.51 & 2.27 & 2.31 \\
\hline & 100 & 2.33 & 2.21 & 2.34 & 2.29 & 2.12 & 2.44 & 2.30 & 1.87 & 2.47 & 2.22 & 2.27 \\
\hline & 50 & 2.35 & 2.22 & 2.37 & 2.31 & 2.17 & 2.44 & 2.32 & 1.88 & 2.45 & 2.23 & 2.29 \\
\hline & 0100 & 2.33 & 2.19 & 2.31 & 2.28 & 2.16 & 2.43 & 2.31 & 1.88 & 2.55 & 2.26 & 2.28 \\
\hline & 1000 & 2.40 & 2.29 & 2.36 & 2.35 & 2.21 & 2.52 & 2.38 & 2.03 & 2.43 & 2.29 & 2.34 \\
\hline & 2000 & 2.37 & 2.36 & 2.29 & 2.34 & 2.13 & 2.46 & 2.32 & 1.92 & 2.48 & 2.25 & 2.31 \\
\hline$=$ & & & & 2.30 & & & 2.32 & & & 2.26 & & \\
\hline$\sum^{\mathbb{N}}$ & & 2.36 & 2.11 & 2.42 & & & & & & & & \\
\hline & $A^{\star} B$ & & 2.24 & 2.31 & & 2.14 & 2.46 & & 1.95 & 2.47 & & \\
\hline & 0.05 & $A ; 0.0$ & & 0.09 & $\mathrm{C} ; 0.16$ & $A^{*} B$ & .16 & $A^{*} C ; 0.27$ & & ;0.27 & $A^{*} B^{*} C$ & $; 0.48$ \\
\hline
\end{tabular}

\begin{tabular}{|c|c|}
\hline N.S.= Nutrient Solution (Control) & SA = Salicylic acid \\
\hline AsA = Ascorbic acid & $\alpha$-toco. $=\alpha$-tocopherol \\
\hline
\end{tabular}

Regarding the promotive effect of $\alpha$-tocopherol on photosynthetic pigments, $\alpha$-tocopherol is located in the chloroplast envelop, thylakoid membranes and plastoglobuli and deactivates photosynthesis derived reactive oxygen species (mainly $1 \mathrm{O} 2$ and $\mathrm{OH}-$ ), which are well protected against photooxidative damage (Munné-Bosch, 2005). In addition, it may have a key role in protection against oxidative stress caused by $\beta$-oxidation; it could be that tocopherol also has this role during senescence as well as it is very abundant in the thylakoid membranes that contain 
polyunsaturated fatty acids and are in close proximity to ROS produced during photosynthesis and correlative evidence strongly suggests an antioxidant role for tocopherol (Munné-Bosch and Sattler et al., 2004).

The stimulatory effect of yeast on chlorophyll and carotenoid in sweet pepper leaves might be due to that the yeast as source of cytokinins which delay the dehydration of chlorophyll via the inhibition of chlorophyllase and enhance the synthesis of protein and RNA that are closely related with delaying the aging of leaves and cytokinins increase number of chloroplasts in the leaf by increasing both intensity of cell growth phytohormones and the activity of cytoplasm ribosomes, thus chlorophyll synthesis is stimulated (Brozenkova and Makrozova, 1976).

\section{Ascorbic acid, proline, total phenols and carbohydrates concentrations:}

Results presented in Tables (6,9 and 10) reveal that the primary components of this system include carotenoids, ascorbic acid, total carbohydrates, proline and total phenols were enhanced with exogenous application of phytohoromnes, vitamins and yeast extract.

The data presented in Tables (9-10) clearly show that increasing salinity levels from 2000 to $4000 \mathrm{mg} / \mathrm{L}$ of all salinity types increased significantly ascorbic acid, proline, total phenols as well as total soluble carbohydrates concentrations in sweet pepper shoot and the highest value was obtained under high salinity level. In addition, sweet pepper plants growing under $\mathrm{NaCl}$ showed a greater increase in ascorbic acid, proline, total phenol as well as total soluble carbohydrates concentrations followed by $\mathrm{NaCl}+\mathrm{CaCl}_{2}$ and $\mathrm{CaCl}_{2}$ as compared to the unstressed plants. Furthermore, pre-soaking seeds in SA, AsA, $\alpha$-tocopherol and yeast extract at both levels increased ascorbic acid, proline, total phenols as well as total soluble carbohydrates concentrations under non-saline conditions. In addition, pre-soaking seeds in AsA at $50 \mathrm{mg} / \mathrm{L}$ and $\mathrm{SA}$ at $75 \mathrm{mg} / \mathrm{L}$ was more effective as compared with the other treatments.

Regarding the interactions, means $\left(A^{*} B\right)$ indicated that ascorbic acid, proline, total phenols as well as total soluble carbohydrates concentrations were significantly increased with increasing salinity level from 2000 to $4000 \mathrm{mg} / \mathrm{L}$ for all salinity types. The maximum increase was recorded for plants grown under $\mathrm{NaCl}$ followed by $\mathrm{NaCl}_{+} \mathrm{CaCl}_{2}$ and $\mathrm{CaCl}_{2}$.

\section{Proline:}

Proline concentration was increased markedly in the leaves of sweet pepper plants with increasing $\mathrm{NaCl}$ and $\mathrm{NaCl}+\mathrm{CaCl}_{2}$ salinity level from 2000 to $4000 \mathrm{mg} / \mathrm{L}$ (Table 9). Plants accumulate proline a non-toxic and protective osmolyte under saline conditions, it is considered to be compatible solutes. Moreover, proline can also confer enzyme protection and increase membrane stability under various conditions. Proline accumulation may also help in nonenzymatic free radical detoxifications (Khan et al., 2002).

Accumulation of proline may be a part of general adaptation to stress recognized as osmotic adjustment agents (Misra and Gupta, 2005) and play a clear role as an osmoticum which can accumulate to high concentrations in the cell cytoplasm without interfering with cellular structure or metabolism (Samaras et al., 1995). These functions include osmoregulation; as a compatible cytoplasmic solute, it apparently counteracts the osmotic potential of the vacuole salts (Bray et al., 2000). Higher osmolytes accumulation especially proline and soluble proteins seems to be related to salt tolerance in sweet pepper as shown in the present investigation and not to be a consequence of tissues reaction to salt stress damage. Various studies have focused on the ability of proline as a compatible osmolytes, which cause the minimal inhibition of metabolism (Siddiqui et al., 2008) and/or enzyme and membrane protection against salt inactivation (Tajdoost et al., 2007).

\section{Phenolic compounds:}

Total phenols in sweet pepper were increased with increasing salinity levels from 2000 to $4000 \mathrm{mg} / \mathrm{L}$ (Table 10). This increase showed some tendency to adjust osmotically against salt stress. These results are in agreement with (Amor et al., 2000), who stated that stress condition leads to an increase in phenolic compounds. These phenolic compounds could be a cellular adaptive mechanism for scavenging oxygen free radicals during stress and this free radical scavenger and others such as ascorbate could be readily oxidized in the system of tissue representing sub-cellular damages.

Until recently, studies of the antioxidant properties of phenolic compounds in vitro, combined with the wellcharacterized activation of phenolic biosynthesis in response to diverse biotic and a-biotic stresses, and has led to a reevaluation of the physiological function of phenolic compounds in plants (Grace and Logan, 2000). 
Table (9): Effect of pre-soaking seeds in SA, AsA, $\alpha$-tocopherol or Yeast extract on ascorbic acid (mg/g FW) and proline concentrations $(\mathrm{mg} / \mathrm{g} \mathrm{DW})$ in sweet pepper shoot grown under non-saline and saline conditions at 75 and 90 days from sowing using NFT.

\begin{tabular}{|c|c|c|c|c|c|c|c|c|c|c|c|c|}
\hline \multirow{3}{*}{\multicolumn{2}{|c|}{$\begin{array}{c}\text { Salinity } \\
(\mathrm{A}) \\
\text { Treatment } \\
\text { (C) } \mathrm{mg} / \mathrm{L}\end{array}$}} & \multirow[b]{3}{*}{ N.S. } & \multicolumn{3}{|c|}{ N.S. $+\mathrm{NaCl}$} & \multicolumn{3}{|c|}{ N.S. $+\mathrm{CaCl}_{2}$} & \multicolumn{3}{|c|}{$\begin{array}{c}\text { N.S. }+\left(\mathrm{NaCl}+\mathrm{CaCl}_{2}\right) \\
(1: 1) \mathrm{w} / \mathrm{w}\end{array}$} & \multirow{3}{*}{$\begin{array}{l}\text { Mean } \\
(\mathrm{C})\end{array}$} \\
\hline & & & \multicolumn{2}{|c|}{ Conc. (B) } & \multirow{2}{*}{$\begin{array}{l}\text { Mean } \\
\left(A^{*} C\right)\end{array}$} & \multicolumn{2}{|c|}{ Conc. (B) } & \multirow{2}{*}{$\begin{array}{l}\text { Mean } \\
\left(A^{*} C\right)\end{array}$} & \multicolumn{2}{|c|}{ Conc. (B) } & \multirow{2}{*}{$\begin{array}{l}\text { Mean } \\
\left(A^{\star} C\right)\end{array}$} & \\
\hline & & & 2000 & 4000 & & 2000 & 4000 & & 2000 & 4000 & & \\
\hline \multicolumn{13}{|c|}{ Ascorbic acid } \\
\hline \multicolumn{2}{|c|}{ Water } & 4.35 & 7.85 & 10.37 & 7.52 & 6.04 & 8.88 & 6.42 & 6.65 & 9.68 & 6.89 & 6.95 \\
\hline \multicolumn{2}{|c|}{ SA 75} & 5.64 & 8.42 & 11.30 & 8.45 & 6.38 & 9.25 & 7.09 & 7.25 & 10.23 & 7.71 & 7.75 \\
\hline \multicolumn{2}{|c|}{ SA 150} & 5.60 & 8.58 & 11.51 & 8.56 & 6.34 & 9.35 & 7.10 & 7.39 & 10.08 & 7.69 & 7.78 \\
\hline \multicolumn{2}{|c|}{ AsA 50} & 5.87 & 8.74 & 11.84 & 8.82 & 6.46 & 9.55 & 7.29 & 7.47 & 10.21 & 7.85 & 7.99 \\
\hline \multicolumn{2}{|c|}{ AsA 100} & 5.99 & 8.81 & 13.69 & 9.50 & 6.51 & 9.62 & 7.38 & 7.55 & 10.27 & 7.94 & 8.27 \\
\hline \multicolumn{2}{|c|}{ a-toco 50} & 5.70 & 8.47 & 11.39 & 8.52 & 6.42 & 9.31 & 7.14 & 7.29 & 10.04 & 7.68 & 7.78 \\
\hline \multicolumn{2}{|c|}{ a-toco 100} & 5.78 & 8.69 & 11.75 & 8.74 & 6.44 & 9.43 & 7.22 & 7.41 & 10.15 & 7.78 & 7.91 \\
\hline \multicolumn{2}{|c|}{ Yeast 1000} & 5.05 & 7.78 & 10.53 & 7.79 & 6.07 & 8.97 & 6.70 & 6.90 & 9.75 & 7.23 & 7.24 \\
\hline \multicolumn{2}{|c|}{ Yeast 2000} & 5.31 & 8.13 & 10.88 & 8.11 & 6.27 & 9.18 & 6.92 & 7.13 & 9.99 & 7.48 & 7.50 \\
\hline \multirow{3}{*}{$\begin{array}{l}\frac{1}{\sqrt{0}} \\
\sum \\
\sum\end{array}$} & & \multirow{3}{*}{5.48} & \multicolumn{3}{|c|}{8.45} & \multicolumn{3}{|c|}{7.03} & \multicolumn{3}{|c|}{7.58} & \\
\hline & & & 7.31 & 10.27 & & & & & & & & \\
\hline & $A^{*} B$ & & 8.39 & 11.48 & & 6.33 & 9.28 & & 7.23 & 10.04 & & \\
\hline & at 0.05 & $A ; 0.1$ & B; & 0.10 & $\mathrm{C} ; 0.17$ & $A^{*} B$ & 0.17 & $A^{*} C ; 0.30$ & & ; 0.30 & $A^{*} B^{*}$ & $; 0.52$ \\
\hline & & & & & & Proli & & & & & & \\
\hline & ater & 3.26 & 5.75 & 7.78 & 5.60 & 4.53 & 6.66 & 4.82 & 4.93 & 7.26 & 5.15 & 5.19 \\
\hline & 75 & 4.49 & 6.61 & 10.27 & 7.12 & 4.89 & 7.22 & 5.53 & 5.66 & 7.70 & 5.95 & 6.20 \\
\hline & 150 & 4.20 & 6.31 & 8.47 & 6.33 & 4.76 & 6.93 & 5.30 & 5.44 & 7.53 & 5.72 & 5.78 \\
\hline & A 50 & 4.40 & 6.55 & 8.88 & 6.61 & 4.84 & 7.16 & 5.47 & 5.60 & 7.67 & 5.89 & 5.99 \\
\hline & 100 & 4.28 & 6.43 & 8.64 & 6.45 & 4.81 & 7.01 & 5.37 & 5.54 & 7.62 & 5.81 & 5.88 \\
\hline & co 50 & 4.23 & 6.35 & 8.55 & 6.38 & 4.78 & 6.98 & 5.33 & 5.47 & 7.56 & 5.75 & 5.82 \\
\hline & 0100 & 4.34 & 6.52 & 8.82 & 6.56 & 4.83 & 7.07 & 5.41 & 5.55 & 7.65 & 5.85 & 5.94 \\
\hline & 1000 & 3.78 & 6.04 & 7.90 & 5.91 & 4.55 & 6.70 & 5.01 & 4.99 & 7.31 & 5.36 & 5.43 \\
\hline & 2000 & 3.98 & 6.17 & 8.12 & 6.09 & 4.66 & 6.88 & 5.17 & 5.27 & 7.45 & 5.57 & 5.61 \\
\hline & & & & 6.34 & 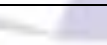 & & 5.27 & & & 5.67 & & \\
\hline$\sum^{\infty}$ & & 4.11 & 5.48 & 7.70 & & & & & & & & \\
\hline & $A^{*} B$ & & 6.30 & 8.60 & & 4.74 & 6.96 & & 5.38 & 7.53 & & \\
\hline & at 0.05 & $A ; 0.0$ & & 0.08 & $\mathrm{C} ; 0.13$ & $A^{*} B$ & 0.13 & $A^{*} C ; 0.22$ & & ; 0.22 & $A^{*} E$ & $; 0.39$ \\
\hline
\end{tabular}

\begin{tabular}{|c|c|}
\hline N.S. = Nutrient Solution (Control) & SA = Salicylic acid \\
\hline AsA $=$ Ascorbic acid & $\alpha$-toco. $=\alpha$-tocopherol \\
\hline Yeast = Yeast extract & \\
\hline
\end{tabular}


Total soluble carbohydrates:

Data presented in Table (10) show that salinity increased total carbohydrates in the shoots of sweet pepper with increasing salinity level from 2000 to $4000 \mathrm{mg} / \mathrm{L}$ as compared to non-salinized plant.

Table (10): Effect of pre-soaking seeds in SA, AsA, $\alpha$-tocopherol or Yeast extract on total phenols (mg/100g FW) and total soluble carbohydrates concentrations $(\mathrm{mg} / \mathrm{g} \mathrm{DW}$ ) in sweet pepper shoot grown under non-saline and saline conditions at 75 and 90 days from sowing using NFT.

\begin{tabular}{|c|c|c|c|c|c|c|c|c|c|c|c|c|}
\hline \multirow{3}{*}{\multicolumn{2}{|c|}{$\begin{array}{c}\text { Salinity } \\
(\mathrm{A}) \\
\text { Treatment }(\mathrm{C}) \\
\mathrm{mg} / \mathrm{L}\end{array}$}} & \multirow[b]{3}{*}{ N.S. } & \multicolumn{3}{|c|}{ N.S.+ $\mathrm{NaCl}$} & \multicolumn{3}{|c|}{ N.S. $+\mathrm{CaCl}_{2}$} & \multicolumn{3}{|c|}{$\begin{array}{c}\text { N.S. }+\left(\mathrm{NaCl}+\mathrm{CaCl}_{2}\right) \\
(1: 1) \mathrm{w} / \mathrm{w}\end{array}$} & \multirow{3}{*}{$\begin{array}{l}\text { Mean } \\
\text { (C) }\end{array}$} \\
\hline & & & \multicolumn{2}{|c|}{ Conc. (B) } & \multirow{2}{*}{$\begin{array}{l}\text { Mean } \\
\left(A^{*} C\right)\end{array}$} & \multicolumn{2}{|c|}{ Conc. (B) } & \multirow{2}{*}{$\begin{array}{l}\text { Mean } \\
\left(A^{*} C\right)\end{array}$} & \multicolumn{2}{|c|}{ Conc. (B) } & \multirow{2}{*}{$\begin{array}{l}\text { Mean } \\
\left(A^{*} C\right)\end{array}$} & \\
\hline & & & 2000 & 4000 & & 2000 & 4000 & & 2000 & 4000 & & \\
\hline \multicolumn{13}{|c|}{ Total phenol } \\
\hline \multicolumn{2}{|c|}{ Water } & 27.18 & 47.93 & 64.80 & 46.64 & 37.77 & 55.51 & 40.15 & 41.11 & 60.50 & 42.93 & 43.24 \\
\hline \multicolumn{2}{|c|}{ SA 75} & 37.45 & 55.07 & 85.59 & 59.37 & 40.71 & 60.15 & 46.10 & 47.19 & 64.18 & 49.60 & 51.69 \\
\hline \multicolumn{2}{|c|}{ SA 150} & 34.96 & 52.62 & 70.61 & 52.73 & 39.62 & 57.78 & 44.12 & 45.32 & 62.74 & 47.67 & 48.18 \\
\hline \multicolumn{2}{|c|}{ AsA 50} & 36.69 & 54.63 & 74.01 & 55.11 & 40.38 & 59.69 & 45.59 & 46.70 & 63.96 & 49.12 & 49.94 \\
\hline \multicolumn{2}{|c|}{ AsA 100} & 35.66 & 53.64 & 71.98 & 53.76 & 40.10 & 58.43 & 44.73 & 46.16 & 63.47 & 48.43 & 48.97 \\
\hline \multicolumn{2}{|c|}{ a-toco 50} & 35.25 & 52.95 & 71.22 & 53.14 & 39.83 & 58.20 & 44.43 & 45.58 & 63.03 & 47.95 & 48.50 \\
\hline \multicolumn{2}{|c|}{ a-toco 100} & 36.15 & 54.32 & 73.47 & 54.65 & 40.25 & 58.93 & 45.11 & 46.28 & 63.78 & 48.74 & 49.50 \\
\hline \multicolumn{2}{|c|}{ Yeast 1000} & 31.55 & 50.33 & 65.81 & 49.23 & 37.96 & 55.82 & 41.78 & 41.56 & 60.92 & 44.68 & 45.23 \\
\hline \multicolumn{2}{|c|}{ Yeast 2000} & 33.16 & 51.39 & 67.68 & 50.74 & 38.81 & 57.35 & 43.11 & 43.91 & 62.10 & 46.39 & 46.75 \\
\hline \multirow{3}{*}{$\begin{array}{l}\frac{1}{\mathbb{ల}} \\
\stackrel{\mathbb{1}}{\Sigma}\end{array}$} & & \multirow{3}{*}{34.23} & \multicolumn{2}{|r|}{52.82} & & \multicolumn{3}{|c|}{43.90} & \multicolumn{3}{|c|}{47.28} & \\
\hline & & & 45.63 & 64.14 & & & & & & & & \\
\hline & $A^{*} B$ & & 52.54 & 71.68 & & 39.49 & 57.98 & & 44.87 & 62.74 & & \\
\hline & tt 0.05 & $A ; 0.6$ & B; & 0.62 & $\mathrm{C} ; 1.08$ & $A^{*} B$ & 1.08 & $A^{*} C ; 1.87$ & $\mathrm{~B}^{*}$ & ; 1.87 & $A^{*} B^{\star}$ & 3.24 \\
\hline & & & & & Total sc & uble car & bohydra & & & & & \\
\hline & ter & 7.28 & 15.11 & 20.87 & 14.42 & 10.96 & 17.36 & 11.87 & 12.32 & 18.81 & 12.81 & 13.03 \\
\hline & 75 & 10.90 & 17.07 & 28.46 & 18.81 & 12.13 & 18.75 & 13.93 & 14.92 & 20.70 & 15.50 & 16.08 \\
\hline & 150 & 10.45 & 16.14 & 23.50 & 16.70 & 11.61 & 18.30 & 13.45 & 13.49 & 19.58 & 14.51 & 14.89 \\
\hline & 50 & 10.79 & 16.72 & 26.19 & 17.90 & 12.04 & 18.64 & 13.82 & 14.60 & 20.51 & 15.30 & 15.68 \\
\hline & 100 & 10.67 & 16.51 & 24.66 & 17.28 & 11.77 & 18.51 & 13.65 & 13.98 & 19.94 & 14.87 & 15.27 \\
\hline & 50 & 10.53 & 16.35 & 24.24 & 17.04 & 11.69 & 18.41 & 13.54 & 13.74 & 19.73 & 14.67 & 15.08 \\
\hline & 0100 & 10.72 & 16.60 & 25.06 & 17.46 & 11.84 & 18.59 & 13.72 & 14.29 & 20.40 & 15.14 & 15.44 \\
\hline & 1000 & 7.92 & 15.58 & 21.83 & 15.11 & 11.02 & 17.61 & 12.18 & 12.39 & 18.91 & 13.07 & 13.45 \\
\hline & 2000 & 9.90 & 15.82 & 22.54 & 16.08 & 11.34 & 18.08 & 13.11 & 12.78 & 19.21 & 13.96 & 14.38 \\
\hline & & & & 16.76 & & & 13.25 & & & 14.42 & & \\
\hline$\stackrel{\infty}{\infty}^{\infty}$ & & 9.91 & 13.81 & 20.72 & & & & & & & & \\
\hline & $A^{*} B$ & & 16.21 & 24.15 & & 11.60 & 18.25 & & 13.61 & 19.75 & & \\
\hline & 0.05 & $A ; 0.0$ & & 0.05 & C; 0.09 & $A^{*} B$ & 0.09 & $A^{*} C ; 0.15$ & & 0.15 & $A^{\star} E$ & $; 0.27$ \\
\hline
\end{tabular}

\begin{tabular}{|c|c|}
\hline AsA = Ascorbic acid & a-toco. $=\alpha$-tocopherol \\
\hline Yeast $=$ Yeast extract & \\
\hline
\end{tabular}


The present results confirm that the carbohydrates content is a very sensitive factor for salt tolerance improvement. The increase in total carbohydrates content in shoots was observed under salinity stress. This was due to an increase in glucose and fructose (reducing sugars) concentration not only in the leaves but also in the roots associated to the presence of high concentration of chloride in the plant tissues (Liu and VanStaden, 2001).

These results indicate a positive relation between high salinity levels and sugar accumulation potential. In addition, glycophytes adapt themselves to somewhat saline conditions by lowering osmotic potential through converting starch to sugar (Larcher, 1995) and accumulation of sugars resulted in decreased oxidative stress because of the radical scavenging features of many solutes (Smirnoff and Cumbes, 1989) and enhanced efficiency in the use of carbon coupled to a reduction in cellular metabolism, that could fervor the accumulation of respiratory substrate to support the osmotic adjustment required to survive in saline media (Schnapp et al., 1990), Moreover, Tajdoost et al. (2007) suggested that the increment in soluble carbohydrate due to salinity may play an important role in increasing the osmotic pressure of the cytoplasm. Furthermore, Bartels and Sunkar (2005) found a strong correlation between sugar accumulation and osmotic tolerance. Hence, improvement of crop performance by increasing osmotic potential-adjusting ability might be more significant in increasing plant growth.

In general, the increment in soluble components among which total sugars due to saline conditions might, in turn, play an important role in increasing the osmotic pressure of the cytoplasm. In addition, sugars as osmolytes enable plants to keep better water relations under salt stress conditions and sugar concentration may be used as an indicator to the osmoprotectant levels in wheat plant and may contribute to salt tolerance in this system. This conclusion is in accordance with the results obtained by Munns et al. (2006) who stated that organic molecules act as osmotica and play an important role in osmotic adjustment in non-halophytes.

It could be concluded that pre-soaking sweet pepper seeds in AsA at $50 \mathrm{mg} / \mathrm{L}$ or SA at $75 \mathrm{mg} / \mathrm{L}$ could alleviate the harmful effect of salinity on the leaf phytochemical contents

\section{REFERENCES}

1. Amor, Y.; Chevion, M. and Levinc, A. 2000. Anoxia pretreatment protects soybean cells against $\mathrm{H}_{2} \mathrm{O}_{2}$-induced cell death: possible involvement of peroxidases and of alternative oxidase. FEBS. Letters, 477 (3): 175-180.

2. Arafa, A.A; Khafagy, M.A. and El-Banna, M.F. 2009. The effect of glycine betaine or ascorbic acid on grain germination and leaf structure of sorghum plants grown under salinity stress. Aust. J. of Crop Sci., 3 (5): 294-304.

3. Bartels, D. and Sunkar, R. 2005. Drought and salt tolerance in plants. Crit. Rev. Plant Sci., 24: 23-58.

4. Bray, E.A.; Bailey-Serres, J. and Weretilnyk, E. 2000. Responses to Abiotic Stress. In Biochemistry and Molecular Biology of Plants (eds: Buchanan B, Gruissem W, Jones, R) Am. Soc. of Plant Biol., Rockville, USA, pp 1158-1203.

5. Brozenkova, R.H. and Morkonzov, H. 1976. The role of phytohormones in biogenesis of chloroplasts. Field Crop Abstract, 32 (4): 2436.

6. Casado-Vela, J.; Sellés, S.; Díaz-Crespo, C.; Navarro-Pedreňo, J.; Mataix-Beneyto, J. and Gómez, I. 2007. Effect of composted sewage sludge application to soil on sweet pepper crop (Capsicum annuum var. annuum) grown under two exploitation regimes. Waste Manag., 27: 1509-1518.

7. Cooper, A. 1979. The ABC of NFT. Growers Books, London, pp. 59.

8. Cumming, R.W. and Elliot, G.L. 1991. Soil chemical properties. In P. E. V. Charman and B. W. Murphy eds., Soils: Their properties and management. Sydney University Press, Melbourne.

9. Epstein El-Banna, M.F. 2006. Comparative studies on structure of some flowering plants as affected by salinity. M.Sc. Thesis, Fac. Agric. Mansoura Univ. Egypt.

10. , E. 1998. How calcium enhances plant salt tolerance. Sci., 40: 1906-1907.

11. FAO, 2008. FAO Land and Plant Nutrition Management Service. http://www.fao.org/ag/agl/agll/spush

12. Gadallah, M.A.A. 2000. Effects of indole-3-acetic acid and zinc on the growth, osmotic potential and soluble carbon and nitrogen components of soybean plants growing under water deficit. J. of Arid Environ., 44: 451-467.

13. Ghallab, A.M. and Nesiem, A.R.A. 1999. Increasing salt tolerance of wheat plants through seed treatment with gamma rays. J. Agric. Sci. Mans. Univ., 24(2): 583-604.

14. Gomez, K.A. and Gomez, A.A. 1984. Statistical Procedures for Agriculture Research. John Wiley and Sons, Inc. New York.

15. Grace, S.C. and Logan, B.A. 2000. Energy dissipation and radical scavenging by the plant phenylpronanoid pathway. Phil. Trans. R. Soc. Lond. B., 355: 1499-1510.

16. Greenway, H. and Munns, R. 1980. Mechanism of salt tolerance in non halophytes. Annu. Rev. Plant Physiol., 31: 149-190.

17. Hare, P.D.; Cress, W.A. and Van Staden, J. 1998. Dissecting the roles of osmolyte accumulation during stress. Plant Cell and Environ., 21: 535-553. 
18. Hatung, W. 2004. Plant response to stress: Abscisic acid fluxes. Marcel Dekker. Inc., New York.

19. Hernandez, J.A.; Jimenez, A.; Mullineaux, P. and Sevilla, F. 2000. Tolerance of pea (Pisum sativum L.) to long-term salt stress is associated with induction of antioxidant defenses. Plant Cell and Environ., 23: 853-862.

20. Jones, M.M. and Turner, T.C. 1978. Osmotic adjustment in leaves of sorghum in response to water deficits. Plant Physiol., 61: 122-126.

21. Kayani, S.A.; Naqvi, H.H. and Ting, I.P. 1990. Salinity effects of germination and mobilization of reserves in jojoba seed. Crop Sci., 30: 704-708.

22. Khan, M.H.; Singha, K.L.B. and Panda, S.K. 2002. Changes in antioxidant levels in Oryza sativa L. roots subjected to $\mathrm{NaCl}$-salinity stress. Acta Physiol. Plant., 24 (2): 145-148.

23. Koca, M.; Bor, M.; Ozdemir, F. and Turkan, I. 2007. The effect of salt stress on lipid peroxidation, antioxidative enzymes and proline content of sesame cultivars. Environ. Exp. Bot., 60: 344-351.

24. Larcher, W. 1995. Physiological Plant Ecology. $3^{\text {rd }}$ Ed. Springer Verlag. Berlin.

25. Li, Y. 2009. Physiological responses of tomato seedlings (Lycopersicon esculentum) to salt stress. Modern Appl. Sci., 3: 171-176.

26. Liu, T. and Van Staden, J. 2001. Growth rate, water relations and ion accumulation of soybean callus lines differing in salinity tolerance under salinity stress and its subsequent relief. Plant Growth Reg., 34: 277-285.

27. Lutts, S.; Kinet J.M. and Bouharmont J. 1996. NaCl-induced senescence in leaves of rice (Oryza sativa L.) cultivars differing in salinity resistance. Ann. of Bot., 78: 389-398.

28. Lycoskoufis, I.H.; Savvas, D. and Mavrogianopoulos, G. 2005. Growth, gas exchange and nutrient status in pepper (Capsicum annuum L.) grown in recirculating nutrient solution as affected by salinity imposed to half of the root system. Scientia Hort., 106: 147-161.

29. Mackiny, G. 1941. Absorption of light by chlorophyll solution. J. Biol. Chem., 140: 315-322.

30. Meneguzzo, S. and Navarilzzo, I. 1999. Antioxidative responses of shoots and roots of wheat to increasing $\mathrm{NaCl}$ concentrations. J. Plant Physiol., 155: 274-280.

31. Misra, N. and Gupta, A.K. 2005. Effect of salt stress on proline metabolic in salt tolerant green gram cultivar. Plant Sci., 169: 331-339.

32. Moharekar, S.T.; Lokhande, S.D.; Hara, T.; Tanaka, R.; Tanaka, A. and Chavan, P.D., 2003. Effect of salicylic acid on chlorophyll and carotenoid contents of wheat and moong seedlings. Photosynthetica, 41: 315-317.

33. Munné-Bosch, S. 2005. The role of a-tocopherol in plant stress tolerance. J. of Plant Physiol., 162: 743-748.

34. Munné-Bosch, S. and Alegre, L. 2002. The function of tocopherols and tocotrienols in plants. Crit. Rev. Plant Sci., 21: 31-57.

35. Munns, R.; James, R.A. and Laüchi, A. 2006. Approaches to increasing the salt tolerance of wheat and other cereals. J. of Exp. Bot., 57: 1025-1043.

36. Nagodawithana, W.T. 1991. Yeast technology. Universal foods cooperation Milwauke, Wisconsin. Published by Van Nostrand, New York, pp. 273.

37. Navarro, J.M.; Flores, P.; Garrido, C. and Martinez, V. 2006. Changes in the contents of antioxidant compounds in pepper fruits at different ripening stages, as affected by salinity. Food Chem., 96: 66-73.

38. Rinaldelli, E. and Mancuso, S. 1996. Response of young mycorrhizal and nonmycorrhizal plants of olive tree (Olea europaea L.) to saline conditions. I. Short term electrophysiological and long term vegetative salt effects. Adv. Hort. Sci., 10: 126-134.

39. Sadasivam, S. and ManicKam, A. 1996. Biochemical methods. Second Ed., New age Int., India.

40. Saha, P.; Chatterjee, P. and Biswas, A. 2010. $\mathrm{NaCl}$ pretreatment alleviated salt stress by enhancement of antioxidant defense system and osmolyte accumulation in mungbean (Vigna radiata L. Wilczek). Ind. J. of Exp. Bio., 48: $593-600$

41. Samaras, Y.; Bressan, R.A.; Csonka, L.N.; Garcia-Rios, M.G.; Paino D’Uizo, M. and Rhodes, D. 1995. Proline accumulation during drought and salinity. IN N. Smirnoff (ef) Environment and plant metabolism: Flexibility and Acclimation. BIOS Scientific pub Oxford, UK. Pp. 161-187.

42. Sankar, B.; Jaleel, C.A.; Manivannan, P.; Kishorekumar, A.; Somasundaram, R. and Panneerselvam, R. 2007. Drought induced biochemical modifications and proline metabolism in Abelmoschus esculentus (L.) Moench. Acta Bot. Croat., 66: 43-56.

43. Santos, C.; Azevedo, A. and Hand Calderia, G. 2001. In Situ and in vitro senescence induced by $\mathrm{KCl}$ stress: nutrition imbalance, lipid peroxidation and antioxidant metabolism. J. Exp. Bot., 52: 351-360. 
44. Sattler, S.E.; Gilliland, L.U.; Magallanes-Lundback, M.; Pollard, M. and Della-Penna, D. 2004. Vitamin E is essential for seed longevity and for preventing lipid peroxidation during germination. Plant Cell, 16:1419-1432.

45. Schnapp, S.R.; Bressan, R.A. and Hasegawa, P.M. 1990. Carbon used efficiency and cell expansion of NaCladapted tobacco cells. Plant Physiol., 93: 384-388.

46. Sharma, P.K. and Hall, D.O. 1991. Interaction of salt stress and photo -inhibition on photosynthesis in barley and sorghum. J. Plant Physiol., 138: 614-619.

47. Shehata, S.A.M.; Saeed, M.A. and Abou EL-Nour, M.S. 2000. Physiological response of cotton plant to the foliar spray with salicylic acid. Ann. Agric. Sci. Ain Shams Univ. 45 (1): 1-18.

48. Siddiqui, M.H.; Khan, M.N.; Mohammed, F. and Khan, M.M.A. 2008. Role of nitrogen and gibberellin $\left(\mathrm{GA}_{3}\right)$ in the regulation of enzyme activities and in osmo -protectant accumulation in Brassica juncea L. under salt stress. $J$. Agron. Crop Sci., 194: 214-224.

49. Smirnoff, N. and Cumbes, Q.T. 1989. Hydroxyl radicals scavenging activity of compatible isolates. Phytochemistry, 28: 1057-1060.

50. Tajdoost, S.; Farboodnia, T. and Heidari, R. 2007. Salt pretreatment enhance salt tolerance in Zea mays L. seedling. Pak. J. Biol. Sci., 10 (12): 2086-2090.

51. Tari, I.; Csiszar, J.; Szalai, G.; Horvath, F.; Pecsvaradi, A.; Kiss, G.; Szepesi, A.; Szabo, M. and Erdei, L., 2002. Acclimation of tomato plants to salinity stress after a salicylic acid pre-treatment. Acta. Biol. Szegediensis, 46: 55-56.

52. Tester, M. and Davenport, R. 2003. $\mathrm{Na}^{+}$tolerance and $\mathrm{Na}^{+}$transport in higher plants. Ann. Bot., 91: 503-527.

53. Troll, W. and Lindsley, J. 1955. A photometric method for the determination of proline. J. Biol. Chem., 215: 655-660.

54. Winston, G.W. 1990. Physio-chemical basis for free radical formation in cells; production and defenses, pp. 57-86. In: R. G. Alscher and J. R. Cummings (eds.). Stress responses in plants. Adaptation and acclimation mechanisms. Wiley-Liss, New York.

55. Wolucka, B.A.; Goossens, A. and Inze, D. 2005. Methyl jasmonate stimulates the de novo biosynthesis of vitamin C in plant cell suspensions. J. of Exp. Bot., 56: 2527-2538.

56. Xiong, L.; Shumaker, K.S. and Zhu, J.K. 2002. Cell signaling during cold, drought and salt stresses. Plant Cell, 14: 165-183. 